\title{
Phacoemulsification and intraocular lens implantation following pars plana vitrectomy: a prospective study
}

Abstract

Aims To examine the intraoperative characteristics of phacoemulsification in eyes that had undergone pars plana vitrectomy and to determine complication rates and visual outcomes.

Methods A prospective study of 45 consecutive cataract operations on vitrectomised eyes performed over a 9-month period by a single surgeon. Data were collected on preoperative characteristics, intraoperative observations and complications, postoperative complications, and visual acuities.

Results Zonular laxity with abnormal deepening of the anterior chamber and mydriasis was noted in six eyes that had undergone extensive vitreous removal for the treatment of retinal detachment or proliferative diabetic retinopathy, but not in eyes that had had limited 'core vitrectomy' such as for macular hole or epiretinal membrane. In two eyes, the initial deepening of the anterior chamber was accompanied by paradoxical shallowing with miosis (the 'infusion deviation syndrome'). Sightthreatening complications were rare.

St Paul's Eye Unit Royal Liverpool University Hospital Prescot Street Liverpool L7 8XP, UK

Correspondence: Mr FG Ahfat Kent County Ophthalmic and Aural Hospital Church Street Maidstone Kent ME14 1DT, UK Tel: + 441622226269 Fax: + 441622226313

E-mail: ahfat99@ hotmail.com
Snellen visual acuity improved in $84.6 \%$ of eyes previously treated for macular hole, $85.7 \%$ of eyes treated for 'macula-on' retinal detachment, $66.7 \%$ of eyes treated for 'maculaoff' retinal detachment, and $57.1 \%$ of eyes treated for diabetic retinopathy.

Conclusions Surgeons need to be aware of additional challenges arising from zonular instability in eyes that have had extensive vitreous removal. A lower infusion bottle height may guard against sudden changes in anterior chamber depth. The use of a corneal tunnel avoids difficulties associated with an unusually deep anterior chamber. Visual
FG Ahfat, CHW Yuen and CP Groenewald

acuity is less likely to improve in patients previously treated for diabetic retinopathy or for macula-off retinal detachment.

Eye (2003) 17, 16-20. doi:10.1038/

sj.eye. 6700232

Keywords: phacoemulsification; pars plana vitrectomy; infusion deviation syndrome

Introduction

Pars plana vitrectomy is now commonly used in the management of various posterior segment disorders ranging from retinal detachment and proliferative diabetic retinopathy to macular hole and epiretinal membrane. ${ }^{1}$ In the phakic eye, pars plana vitrectomy often results in cataract formation. ${ }^{2-4}$ Cataract surgery in the vitrectomised eye has been reported to present special challenges, which include the loss of vitreous support, unstable posterior capsules, weakened zonules, and posterior capsular plaques. ${ }^{5-8}$ In this study, we examine the intraoperative characteristics of phacoemulsification in the vitrectomised eye, associated complications, and evaluate the visual outcomes.

\section{Materials and methods}

We carried out a prospective study of 45 consecutive cataract operations performed on vitrectomised eyes. All surgery was carried out by a single surgeon (FGA) on a vitreoretinal firm over a 9-month period. Datasets included the patient's age, past ocular history, preoperative visual acuity, intraoperative observations and complications, postoperative complications, the need for neodyniumyttrium-aluminium-garnet (Nd:YAG) laser posterior capsulotomy for residual posterior 
capsule plaque, and corrected visual acuity 6 weeks postoperatively.

Cataract surgery was performed under monitored retrobulbar local anaesthesia in 39 patients, using approximately $3.5 \mathrm{ml}$ of $2 \%$ lignocaine administered by the surgeon and without ocular compression. Six patients had surgery under general anaesthesia. In all eyes, a superior corneal incision was employed, balanced salt solution supplemented with adrenaline was used as irrigating fluid, and Viscoat (sodium chondroitin sulphate/sodium hyaluronate) as viscoelastic. A continuous curvilinear capsulorhexis was achieved in all eyes and hydrodissection was performed with a Bolger hydrodissecting canula. The phacoemulsification technique consisted of a standard divide-and-conquer method (36 eyes) or of phacoaspiration in eyes with a soft nucleus (nine eyes). We used an Alcon phacoemulsifier (Legacy 20000 Series or Accurus) (Alcon Labs, Fort Worth, TX, USA) with a $30^{\circ}$ and $0.9 \mathrm{~mm}$ Turbosonics microtip. The phacoemulsification settings were $20 \mathrm{ml} / \mathrm{min}$ aspiration rate, $15 \mathrm{mmHg}$ vacuum limit for sculpting, and $100 \mathrm{mmHg}$ for the removal of fragments at an initial bottle height of $65-70 \mathrm{~cm}$. These settings were altered if abnormal fluctuations in the anterior chamber depth were observed during phacoemulsification. For example, the bottle height would be increased or the vacuum limit decreased if anterior chamber shallowing was noted. A foldable acrylic implant (Acrysof MA30, Alcon) was used in all eyes, except in those requiring sulcus fixated lens or anterior chamber lens, where a rigid polymethylmethacrylate (PMMA) intraocular implant was used. A number of patients had planned removal of silicone oil at the time of cataract extraction. In these eyes, a posterior capsulotomy was fashioned and silicone oil was aspirated with a Peyman cannula prior to the insertion of a lens implant.

\section{Results}

The age of the patients ranged from 22.6 to 80.2 years with a mean of 64.1 years. The indications for vitrectomy were retinal detachment (19 patients), macular hole (13 patients), proliferative diabetic retinopathy (seven patients), epiretinal membrane (five patients), and retinal vein occlusion (one patient). In all, 35 patients (77.8\%) had received intraocular tamponade with expansile gas or silicone oil. A total of 34 patients had undergone one vitreoretinal procedure prior to the cataract surgery, 10 patients had undergone two procedures, and one patient had undergone three procedures. The median time from the first vitrectomy to cataract surgery was 15.8 months (range 7.2-46.7 months). Six eyes also underwent planned removal of silicone oil during cataract surgery.

\section{Intraoperative observations and complications}

Table 1 summarises the intraoperative observations and complications encountered. An abnormal deepening of the anterior chamber was noted in six eyes. Characteristically, the iris-lens diaphragm bowed posteriorly as soon as irrigation was commenced, causing the anterior chamber to deepen excessively and the pupil to dilate widely. The abnormal anterior chamber configuration persisted when the infusion bottle was lowered and the vacuum settings of the phacoemulsification unit altered. The deepening of the anterior chamber necessitated steeper angulation of the phaco probe during nuclear sculpting. The nucleus was also noted to be more mobile than usual during sculpting. Of these six eyes, two displayed rapid and unpredictable anterior and posterior excursions of the iris-lens diaphragm during phacoemulsification. In these patients, the initial deepening of the anterior chamber was accompanied by sudden shallowing or collapse, as well as pupillary miosis. This was particularly evident when the phaco probe was positioned deep to the iris plane. However, checks on the wound, side port, infusion lines, and on the fluid dynamics of the phacoemulsification unit did not reveal a cause for the sudden fluctuations in the anterior chamber depth. Increasing the height of the infusion bottle appeared to exacerbate rather than correct the shallowing of the anterior chamber, which was eventually managed by injecting viscoelastic material into the eye. We noted that five of these eyes had had two or more vitreoretinal procedures either for retinal detachment or for diabetic vitreous haemorrhage, and one eye was highly myopic and had undergone one vitrectomy procedure for retinal detachment. No such difficulties were encountered in patients who had been treated for macular holes or for

Table 1 Observations and complications in 45 eyes

\begin{tabular}{ll}
\hline Intraoperative & \\
Abnormally deep anterior chamber & $6(13.3 \%)$ \\
Shallow anterior chamber ('infusion deviation & $2(4.4 \%)$ \\
syndrome') & \\
Zonular dehiscence & $1(2.2 \%)$ \\
Posterior capsular plaque & $6(13.3 \%)$ \\
& \\
Postoperative & \\
Transient corneal oedema & $2(4.4 \%)$ \\
Transient raised intraocular pressure & $2(4.4 \%)$ \\
Retinal detachment & $1(2.2 \%)$ \\
Posterior capsular opacification needing & $1(2.2 \%)$ \\
Nd:YAG laser &
\end{tabular}


epiretinal membranes. The type of anaesthetic did not appear to have an influence on the fluctuation of the anterior chamber depth. Of the six patients who displayed the phenomenon, five had surgery under retrobulbar local anaesthesia and one had a general anaesthetic. We used two different Alcon phacoemulsification machines, as the surgery was shared between two operating theatres. However, in the author's experience, the two machines did not appear to differ significantly or to have an influence on the intraoperative observations.

No posterior capsular rupture was recorded, but surgery was complicated by inferior zonular dehiscence in one patient who had previously been treated for a vitreous haemorrhage secondary to diabetic retinopathy. All lens material was successfully removed and an anterior chamber lens implant was inserted.

Posterior capsular plaques were noted in six eyes, and were successfully removed in five eyes with an irrigating Rycroft cannula and a capsule polisher. A total of 38 eyes received foldable acrylic lenses placed within the capsular bag. In six eyes that had simultaneous removal of silicone oil, the section was enlarged, a rigid PMMA implant (IOLAB 6840B) was inserted in the sulcus, and the eye was closed with three interrupted 10/0 nylon sutures. An anterior chamber implant was used in one patient where surgery had been complicated by zonular dehiscence.

\section{Postoperative complications and outcome}

One patient developed a recurrence of his retinal detachment 1 week after cataract surgery combined with removal of silicone oil. He declined further surgical intervention. Transient corneal oedema was noted in two eyes, and raised intraocular pressure in two eyes. Fundus fluorescein angiograms were not performed routinely, but we found no cases of cystoid macular oedema on slitlamp biomicroscopy. Nd:YAG laser posterior capsulotomy was carried out on one eye for a persistent capsular plaque.

Figure 1 summarises the pre- and postoperative visual acuities. Snellen acuity improved in 34 eyes $(75.6 \%)$, was unchanged in nine eyes (20.0\%), and deteriorated in two eyes $(4.4 \%)$. The two eyes that showed deterioration in visual acuity comprised one with a recurrence of retinal detachment after cataract surgery combined with removal of silicone oil and one with diabetic maculopathy. Table 2 summarises the changes in visual acuity after cataract surgery in the various patient subgroups. Visual acuity improved in $84.6 \%$ of patients previously treated for macular hole and in $80.0 \%$ of patients who had had surgery for epiretinal membrane. In contrast, only $57.1 \%$ of patients with diabetic

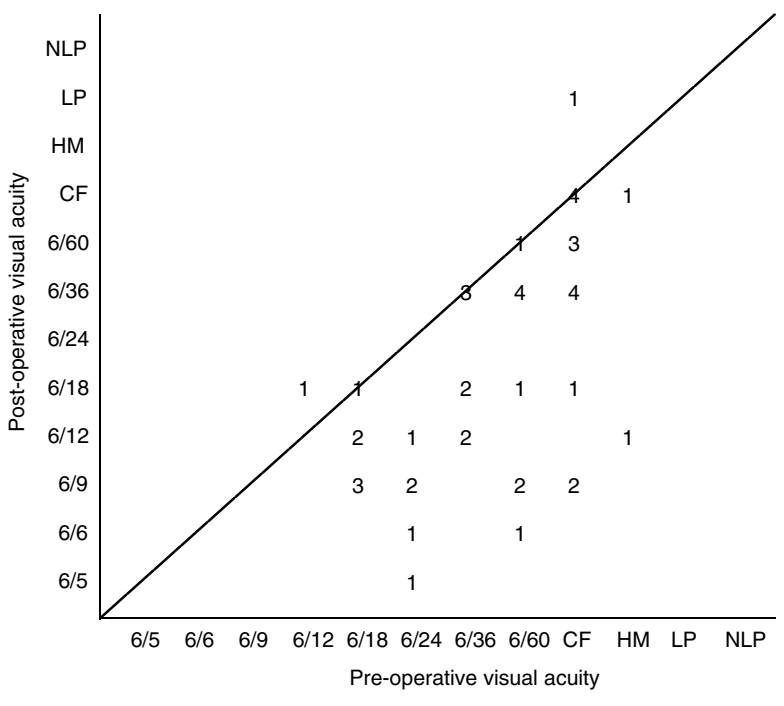

Figure 1 Scattergram of postoperative visual acuity vs preoperative visual acuity: $\mathrm{CF}$, counting fingers; $\mathrm{HM}$, hand motions; LP, light perception; NLP, no light perception.

retinopathy showed improvement in visual acuity after cataract surgery $\left(P=0.22, \chi^{2}\right.$ test). For patients who had undergone vitrectomy for retinal detachment, improvement in visual acuity was noted in $85.7 \%$ of patients with previous 'macula-on' retinal detachment, but in only $66.7 \%$ of patients with 'macula-off' retinal detachment. However, the number of patients was too small for the difference to reach statistical significance $\left(P=0.36, \chi^{2}\right.$ test $)$.

\section{Discussion}

Cataract formation is common after pars plana vitrectomy. With the ever-widening application of vitrectomy in the treatment of many posterior segment disorders, one can expect an increase in the number of such cataracts being referred to general ophthalmologists and anterior segment surgeons. Difficulties with nucleus expression extracapsular cataract extraction have been reported in the vitrectomised eye, and have been presumed to be caused by the loss of vitreous counter pressure. ${ }^{5,6}$ With phacoemulsification, abnormal variations in the anterior chamber depth, unstable zonules, and posterior capsules have also been described. McDermott $\mathrm{et} \mathrm{al}^{7}$ reported on 22 eyes that had vitrectomy for proliferative diabetic retinopathy, retinal detachment, macular holes, and epiretinal membranes. They found abnormal deepening of the anterior chamber in almost all of their patients during phacoemulsification, as well as flaccid posterior capsules during cortical aspiration. However, Grusha et $a l^{8}$ reported an incidence of only 
Table 2 Visual outcomes of cataract surgery

\begin{tabular}{lcccc}
\hline & \multirow{2}{*}{$\begin{array}{c}\text { Number } \\
\text { of patients }\end{array}$} & & Snellen visual acuity \\
\cline { 3 - 5 } & & Improved & Unchanged & Worsened \\
\hline 'Macula-on' retinal detachment & 7 & $6(85.7 \%)$ & $1(14.3 \%)$ & 0 \\
'Macula-off' retinal detachment & 12 & $8(66.7 \%)$ & $3(25.0 \%)$ & $1(8.3 \%)$ \\
Macular hole & 13 & $11(84.6 \%)$ & $2(15.4 \%)$ & 0 \\
Diabetic retinopathy & 7 & $4(57.1 \%)$ & $2(28.6 \%)$ & $1(14.3 \%)$ \\
Epiretinal membrane & 5 & $4(80.0 \%)$ & $1(20.0 \%)$ & 0 \\
Retinal vein occlusion & 1 & $1(100 \%)$ & 0 & 0 \\
\hline
\end{tabular}

$4.5 \%$ of fluctuations in anterior chamber depth during phacoemulsification on 43 vitrectomised eyes. ${ }^{8}$ Several explanations have been proposed for these phenomena, and range from the loss of the vitreous scaffold to the stretching of the zonules by expansile gas and damage to the zonular apparatus during vitrectomy. ${ }^{7-9}$ Our study suggests that these changes are more likely to be encountered in eyes that have had multiple or extensive vitrectomies for retinal detachment or proliferative diabetic retinopathy. No difficulties were encountered in our patients who had limited 'core vitrectomy' with or without gas tamponade for macula hole or for epiretinal membrane. Of the six patients noted to display abnormal anterior chamber deepening and zonular laxity, five had undergone two or more vitrectomies for retinal detachment or proliferative diabetic retinopathy and one patient was a high myope who had been treated for a retinal detachment. These patients were likely to have had extensive vitreous removal through deep indentation and trimming of the vitreous base, either to relieve anterior vitreous traction or to clear persisting vitreous haemorrhage. It is likely that these manoeuvres may have led to structural changes in the region of the vitreous base, resulting in abnormal laxity of the zonules. We did not observe any normalisation of the anterior chamber depth from decreasing the bottle height or from changing the vacuum settings of the phacoemulsification unit. We observed two instances of the so-called 'infusion deviation syndrome' where the initial deepening of the anterior chamber was followed by sudden and unpredictable shallowing. ${ }^{10}$ Both eyes had undergone two vitrectomies for diabetic vitreous haemorrhage and for retinal detachment, respectively. Paradoxically, increasing the bottle height so as to improve fluid inflow only led to further shallowing or collapse of the anterior chamber.

Abnormal anterior chamber deepening and the 'infusion deviation syndrome' probably represent variations of the same phenomenon. In the normal eye, the vitreous acts as a 'clamp' that limits the lensdiaphragm excursions. In the vitrectomised eye however, the absence of this 'clamp' may cause exaggerations in the lens-diaphragm excursions, especially if there has been extensive vitreous removal or if there is significant postocclusion surge and flow changes. Some vitrectomised eyes may also have localised zonular defects that may lead to fluid being exchanged between the anterior chamber and the vitreous cavity. As the phaco tip is inserted, some fluid may be lost both from the anterior chamber and the vitreous cavity. However, as irrigation is commenced, the anterior chamber deepens, owing to reduced vitreous resistance and to the fact that fluid is being directed anterior to the iris plane. This causes the iris to bow posteriorly against the lens, leading to an increase in the pupil size. When phacoemulsification is commenced, the phaco tip is positioned posterior to the iris plane. This may result in fluid being directed through potential zonular defects into the vitreous cavity, causing increased pressure posteriorly and hence anterior chamber shallowing. Increasing the bottle height only serves to increase the fluid inflow into the vitreous cavity and results, paradoxically, in further anterior chamber collapse. In the authors' experience, this 'infusion deviation syndrome' can be minimised by using a lower bottle height, by keeping the phaco tip anterior to the iris plane, and by avoiding in-the-bag phacoemulsification if abnormal fluctuations of the anterior chamber depth are encountered. When excessive shallowing of the anterior chamber is encountered, we suggest that the bottle height is not raised, but instead that the anterior chamber is reformed with viscoelastic material.

All our patients had phacoemulsification through a corneal tunnel. We feel that the possible need for a steeper angulation of the phacoemulsification probe when operating on the vitrectomised eye makes this preferable to a scleral tunnel. The use of a corneal tunnel also avoids the conjunctival scarring or vascularised episclera that is often observed in these eyes. Previous authors have advocated avoiding corneal tunnels in diabetic patients owing to concerns over an increased risk of delayed healing and epithelial complications. ${ }^{7,8,11,12}$ 
However, we found no instances of wound leaks, delayed healing, or persistent epithelial defects in our diabetic patients.

Visual acuity improved in $75.6 \%$ of eyes overall, but varied in the different subgroups of patients.

Improvement in visual acuity after cataract surgery is dependent on a number of factors, which include preoperative visual acuity, the extent of lens opacities, intra- and postoperative complications, and the presence of pre-existing ocular pathology. Patients with more extensive macular pathology, in particular, are less likely to experience significant improvement in vision, irrespective of the extent of lens opacities. In our study, the majority of patients who had had surgery for macula-on retinal detachment, macular hole, and epiretinal membrane experienced improvement in visual acuity after cataract surgery. In contrast, visual improvement was less commonly seen in patients previously treated for macula-off retinal detachment or for proliferative diabetic retinopathy. These patients are more likely to have impaired macular function, and should therefore be given a guarded prognosis for cataract surgery.

In conclusion, this study highlights the additional difficulties that may present during cataract surgery in eyes that have had extensive vitreous removal for retinal detachment or for proliferative diabetic retinopathy. Improvement in visual acuity is seen in most patients, but tends to be more limited in patients with diabetic retinopathy or with previous macula-off retinal detachment.

\section{References}

1 Ah-Fat FG, Sharma MC, Majid MA et al. Trends in vitreoretinal surgery at a tertiary referral centre: 1987 to 1996. Br J Ophthalmol 1999; 83: 396-398.

2 Novak MA, Rice TA, Michels RG et al. The crystalline lens after vitrectomy for diabetic retinopathy. Ophthalmology 1984; 91: 1480-1484.

3 de Bustros S, Thompson JT, Michels RG et al. Nuclear sclerosis after vitrectomy for idiopathic epiretinal membranes. Am J Ophthalmol 1988; 105: 160-164.

4 Melberg NS, Thomas MA. Nuclear sclerotic cataract after vitrectomy in patients younger than 50 years of age. Ophthalmology 1995; 102: 1466-1471.

5 Sneed S, Parrish RK, Mandelbaum S et al. Technical problems of extracapsular cataract extractions after vitrectomy. Arch Ophthalmol 1986; 104: 1126-1127.

6 Smiddy WE, Stark WJ, Michels RG et al. Cataract extraction after vitrectomy. Ophthalmology 1987; 94: 483-487.

7 McDermott ML, Puklin JE, Abrams GW et al. Phacoemulsification for cataract following pars plana vitrectomy. Ophthal Surg Lasers 1997; 28: 558-564.

8 Grusha YO, Masket S, Miller KM. Phacoemulsification and lens implantation after pars plana vitrectomy. Ophthalmology 1998; 105: 287-294.

9 Lacalle VD, Garate FJO, Alday NM et al. Phacoemulsification cataract surgery in vitrectomized eyes. J Cataract Refract Surg 1998; 24: 806-809.

10 Nichamin LD. Phacoemulsification following vitreoretinal surgery. In: Lu LW, Fine IH (eds). Phacoemulsification in Difficult and Challenging Cases. Thieme: New York, 1999, pp 145-150.

11 Schultz RO, van Horn DL, Peters MA et al. Diabetic keratopathy. Trans Am Ophthalmol Soc 1981; 79: 180-199.

12 Baer RM, Aylward WG, Leaver PK. Cataract extraction following vitrectomy and silicone oil tamponade. Eye 1995; 9: $309-312$. 